\title{
Rosuvastatin-Induced Acute Interstitial Nephritis
}

\author{
Francesco Londrino $^{\mathrm{a}} \quad$ Tito Zattera $^{\mathrm{a}}$ Valeria Falqui ${ }^{\mathrm{a}}$ Valentina Corbani ${ }^{\mathrm{a}}$ \\ Marco Cavallini ${ }^{\mathrm{a}}$ Teseo Stefanini ${ }^{\mathrm{b}} \quad$ Nadia Chiappini $^{\mathrm{a}} \quad$ Michela Ardini $^{\mathrm{a}}$ \\ Valentina Martina $^{a}$ Giuseppe Rombolà ${ }^{a}$
}

Departments of ${ }^{a}$ Nephrology and Dialysis and ${ }^{b}$ Radiology, Ospedale S. Andrea, La Spezia, Italy

\section{Key Words}

Rosuvastatin · Acute interstitial nephritis · Acute kidney injury

\begin{abstract}
We report a case of acute interstitial nephritis (AIN), most likely induced by rosuvastatin, in an 83-year-old male patient. The patient underwent angioplasty of the left internal carotid artery, after which he began a regimen of rosuvastatin ( $20 \mathrm{mg} /$ day). After 3 weeks the patient was admitted to our unit for acute renal failure with mild proteinuria with negligible urinary sediment. A left kidney biopsy showed dense interstitial infiltrates, mainly composed of lymphocytes with evident tubulitis. Rosuvastatin withdrawal plus prednisolone ( $1 \mathrm{mg} / \mathrm{kg} /$ day) treatment, which was slowly tapered over a period of 4 weeks, allowed for a complete recovery of renal function. To our knowledge, this is the first case report of rosuvastatininduced AIN. Acute renal failure is associated with a clear increase in morbidity, length of hospital stay and mortality. Moreover, since statins are among the most widely prescribed drugs in Western countries, we think that the risk of AIN should be taken into account as a possible side effect of rosuvastatin.
\end{abstract}

\section{Introduction}

Drug-induced acute interstitial nephritis (AIN) represents a well-known cause of acute kidney injury. The literature reports that AIN is most commonly induced by either antibiotics or anti-inflammatory drugs, with no reports having been previously published where statins may be the cause of AIN. 
The currently known adverse effects of rosuvastatin range from myalgia (3-13\%), headache, dizziness, nausea, abdominal pain, constipation, increased ALT, arthralgia and increased CPK (2-10\%) to serious although uncommon events including new-onset diabetes mellitus, proteinuria, rhabdomyolysis, hypersensitivity and other complications (1-2\%) [1, 2]. In this study we report a case of biopsy-proven AIN most likely induced by rosuvastatin.

\section{Case Report}

An 83-year-old man was admitted to our unit for rapidly worsening renal function 3 weeks following angioplasty of the left internal carotid artery. During this 3-week period his serum creatinine increased from 2.0 to $5.4 \mathrm{mg} / \mathrm{dl}$. The patient had suffered from hypertension within the last 20 years and had chronic kidney disease with unremarkable urinalysis and diffuse peripheral vasculopathy. At the time of admission, he was being treated with an angiotensin receptor blocker (ARB), a dihydropyridine calcium channel blocker, aspirin and rosuvastatin $(20 \mathrm{mg} /$ day $)$, which was prescribed following the carotid angioplasty. At admission the patient was afebrile, eupneic and mildly hypovolemic, showed normal diuresis and had a blood pressure of $110 / 60 \mathrm{~mm} \mathrm{Hg}$ and a heart rate of $88 \mathrm{bpm}$. Laboratory tests were normal except for elevated serum creatinine $(5.4 \mathrm{mg} / \mathrm{dl})$ and BUN $(73 \mathrm{mg} / \mathrm{dl})$. Urinalysis showed no active sediment with mild proteinuria $(500 \mathrm{mg} /$ day). ANA, ENA, C3, C4, CH50, ANCA, cryoglobulinemia, rheumatoid factor, serum and urine protein immunofixation and free light-chain concentrations were all within the normal range. Kidney ultrasonography showed elevated echogenicity of the parenchyma and an increased renal resistance index (0.76) in the absence of renal artery stenosis. The ARB was discontinued and the patient received intravenous crystalloids for the next $72 \mathrm{~h}$. At this time, the possibility of atheroembolic renal disease was excluded since the recent carotid angioplasty was performed through a femoral artery catheter, during which both an ophthalmologic examination for retinal cholesterol crystal emboli and dermatological manifestations were negative and did not show eosinophilia. However, because renal function did not recover (serum creatinine: $5.9 \mathrm{mg} / \mathrm{dl}$ ), an echo-guided biopsy of the left kidney was performed. Light microscopy showed interstitial edema with lymphocyte infiltrate associated with tubulitis (fig. 1). Electron microscopy evaluation was negative. Immunofluorescence was aspecific and not consistent. Based on these results, a diagnosis of acute tubulointerstitial nephritis was made.

Since rosuvastatin was the most recently introduced drug, a possible relationship between the drug and AIN was considered. Thus, rosuvastatin was withdrawn, and oral prednisolone $(1 \mathrm{mg} / \mathrm{kg} /$ day) was prescribed but rapidly tapered over a period of 4 weeks. The patient was discharged with conservative therapy, and after 8 weeks renal function values returned to baseline (serum creatinine $2 \mathrm{mg} / \mathrm{dl}$, BUN $30 \mathrm{mg} / \mathrm{dl}$, proteinuria 150 $\mathrm{mg} / 24 \mathrm{~h}$ and urinalysis at polarized light microscopy was unremarkable).

One month later, the same ARB was reintroduced and, because of the high cardiovascular risk of the patient, a different statin (atorvastatin $20 \mathrm{mg} /$ day) was prescribed. The patient is being regularly followed up at our outpatient service and, 6 months after the beginning of atorvastatin, has not shown any deterioration in renal functions. 


\section{Discussion}

AIN represents a well-known cause of acute kidney injury, which is in turn associated with increased morbidity, length of hospital stay and mortality. Moreover, acute kidney injury, even in the presence of a complete recovery of renal function, may increase the probability that a preexisting moderate renal failure may progress to end-stage renal disease. Together with autoimmune disorders, infections, sarcoidosis and tubulointerstitial nephritis and uveitis (TINU) syndrome, drugs such as antibiotics and anti-inflammatory agents are the most common causes of AIN in adults.

Previously to this report, there has been no available data regarding the possibility of rosuvastatin-induced AIN. In order to demonstrate the association between this drug and interstitial nephritis, 2 important criteria have to be met: (1) the absence of other drugs that may induce AIN, and (2) a kidney biopsy demonstrating that AIN is effectively the cause of the observed acute renal impairment. Given the temporal relationship between the initiation of rosuvastatin intake and the occurrence of acute renal failure, combined with the exclusion of other possible causes, it was believed that the adverse event reported by our patient could be directly related to rosuvastatin treatment, a diagnosis that was subsequently confirmed by renal biopsy.

Statins are among the most widely prescribed drugs in Western countries, and despite the millions of people exposed to statins for several years, there are no reports of such side effect. Thus, statin-induced AIN may be exceptionally rare, or instead, it may be specifically related to rosuvastatin. In this case the probability to have AIN is still rare, but much less than would have been expected if it were a class effect. The statin class consists of a number of heterogeneous drugs which vary in properties such as potency in lowering low-density lipoprotein cholesterol levels, lipophilicity, renoprotection, increasing high-density lipoprotein cholesterol levels, lowering triglyceride levels, and effects on glucose metabolism and myocardial function. Rosuvastatin induced renal side effects, such as microhematuria and worsening of the proteinuria, and deterioration of renal function has been reported [3]. Moreover, the evidence that a different statin (atorvastatin) prescribed to the patient and a subsequent observation follow-up for more than 6 months did not induce any deterioration of renal function and/or increase of proteinuria suggests that in our patient rosuvastatin may be directly involved in AIN [4]. This observation might be relevant in the near future with the introduction of newer statins and/or the combination with other drugs such as polypills.

\section{Acknowledgements}

We thank Dr. Michele Moroni (St. Andrea Hospital, La Spezia) for his assistance.

\section{Disclosure Statement}

The authors have no conflicts of interest to disclose. 
Londrino et al.: Rosuvastatin-Induced Acute Interstitial Nephritis

\section{References}

1 Bays H: Statin safety: an overview and assessment of the data - 2005. Am J Cardiol 2006; 97:6C-26C.

2 FDA Advisory Committee Meeting: CRESTOR (ZD4522, rosuvastatin calcium) TABLETS. Briefing Document NDA 21-366 for the Use of CRESTOR. 2003; US Food and Drug Administration, www.fda.gov.

-3 Agarwal R: Statin induced proteinuria: renal injury or renoprotection? J Am Soc Nephrol 2004;15:25022503.

4 Bianchi S, Bigazzi R, Caiazza A, Campese VM: A controlled, prospective study of the effects of atorvastatin on proteinuria and progression of kidney disease. Am J Kidney Dis 2003;41:565-570.

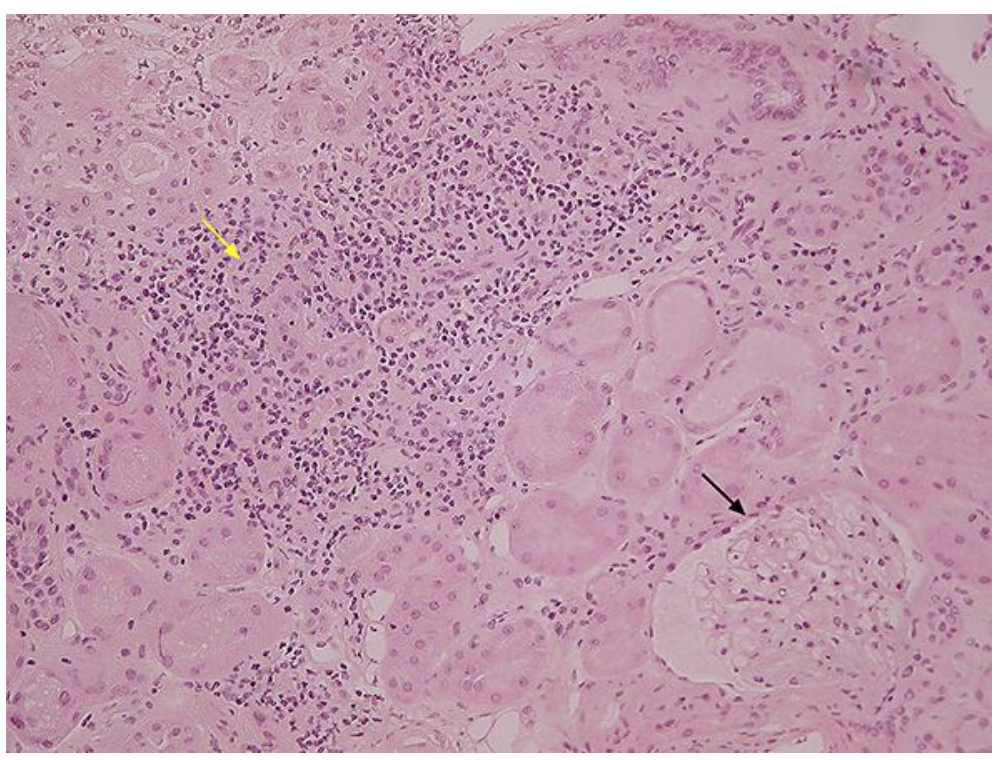

Fig. 1. Light microscopy with hematoxylin and eosin stain showing AIN with diffuse interstitial infiltrate and many lymphocytes associated to tubulitis (yellow arrow) in the presence of an uninvolved glomerulus (black arrow). 